\title{
A methodology for simulating biological systems using Microsoft Excel
}

\author{
Angus M. Brown * \\ Department of Neurology, Box 356465, University of Washington School of Medicine, Seattle, WA 98195-6465, USA
}

Received 28 August 1997; received in revised form 8 June 1998; accepted 23 June 1998

\begin{abstract}
The objective of this present study was to develop a simple, easily understood methodology for solving biologically based models using a Microsoft Excel spreadsheet. The method involves the use of in-cell formulas in which Rows and Columns of new data are generated from data typed into the spreadsheet, but does not require any programming skills or use of the macro language. The approach involves entering the key parameter values into the spreadsheet and conducting the simulation by solving a set of equations based on these parameter values. The examples used in this paper are firstly, a simple voltage clamp simulation in which initial parameter values are used to calculate a system in steady state. The second example is a current clamp simulation where steady state is not reached and the solution of the equations for each time increment is used as the input for the next time increment in the simulation. The calculations are based on the Hodgkin Huxley mathematical equations that describe the voltage dependence of ion channel behavior. The problems and flexibility of the method are briefly discussed. The methodology developed in this present study should help novice modelers to create simple simulations without the need to learn a programming language or purchase expensive software. (C) 1999 Elsevier Science Ireland Ltd. All rights reserved.
\end{abstract}

Keywords: Microsoft Excel; Simulation; Modeling; Hodgkin Huxley; Ion channel

\section{Introduction}

This article is aimed at biologists who wish to simulate or model biological phenomena. A model is a set of equations derived from experimental observations that predict a fundamental

* Tel: + 1206 6168278; fax: +1 206 6858100; e-mail: ambrown@u.washington.edu biological system, the better the model the more accurately it describes the system. For biologists who wish to carry out such modeling there are a number of choices. The most obvious is to write a program in a programming language such as $\mathrm{C}+$ + or Basic as it has the advantage of being specifically written for its intended purpose. Disadvantages include that writing a program requires both the purchase of the program and the 


\begin{tabular}{|c|c|c|c|c|c|}
\hline & $\mathbf{A}$ & B & C & D & $\mathbf{E}$ \\
\hline 1 & 5 & $=A 1^{\wedge} 2$ & 1 & $=C 1^{\wedge} 2$ & $=\$ C \$ 1^{\wedge} 2$ \\
\hline 2 & & & 2 & $=C 2^{\wedge} 2$ & $=\$ C \$ 1^{\wedge} 2$ \\
\hline 3 & & & 3 & $=\mathrm{C3}^{\wedge}{ }^{\wedge}$ & $=\$ C \$ 1^{\wedge} 2$ \\
\hline 4 & & & 4 & $=C 4^{\wedge} 2$ & $=\$ C \$ 1^{\wedge} 2$ \\
\hline 5 & & & 5 & $=\mathrm{C5}^{\wedge} \mathrm{2}$ & $=\$ C \$ 1^{\wedge} 2$ \\
\hline 6 & & & 6 & $=C 6^{\wedge} 2$ & $=\$ C \$ 1^{\wedge} 2$ \\
\hline 7 & & & 7 & $=C 7^{\wedge} 2$ & $=\$ C \$ 1^{\wedge} 2$ \\
\hline 8 & & & 8 & $=C 8^{\wedge} 2$ & $=\$ C \$ 1^{\wedge} 2$ \\
\hline
\end{tabular}

Fig. 1. Spreadsheet used to illustrate features of Excel that allow simulations to be conducted. Columns are lettered and run horizontally and Rows are numbered and run vertically (see text for details).

time to learn to program, skills that many biologists do not have and which are very time consuming. In DOS-based BASIC programs such as Qbasic or GW-Basic it took a couple of hours to acquire the knowledge necessary to produce a simple working ten line program. Today's Windows based environment require the user to understand both the structure and implementation of Object Orientated Programming as well as the increasingly complicated programming languages before a program can be written. In addition debugging programs can be very time consuming, requiring up to half the development time of the program [1]. A more attractive proposition is to use a software program written for the purpose. There are several programs available including Neurosim [2], Neuron [3] and Genesis [4], which are ideally suited to the task of modeling the examples described in this study. Neurosim is aimed at students and is the program most suitable to the simulations described in this paper. Its advantages include that it is designed to help teach neurophysiology at the under- and graduate level and it has been written with ease of use as a primary feature. It runs on a desktop PC but it is expensive. Other programs have the attraction of being free but this is offset by the fact that as their target users are experienced modelers, the programs tend to have obtuse documentation, difficult to comprehend interfaces and may require a computing environment (UNIX) beyond the resources of most biologists. The methodology described in this study offers a cheap alternative. Spreadsheets are among the most commonly used software and most biologists have experience of using them even if at an elementary level. The features of Excel which make it ideal for these purposes are a friendly user interface, flexible data manipulation, in-built mathematical functions and instantaneous charting of data. The objective of the present study was to develop a methodology for solving simple biological models using Microsoft Excel.

\section{Computational method}

The approach used to conduct the simulation involved writing out the equations that describe the Hodgkin Huxley model of voltage gated channel activation (see Appendix), keying the parameter values and equations into the spreadsheet and conducting the simulation by solving the equations.

\subsection{Elementary properties of spreadsheets}

Fig. 1 illustrates elementary properties that allow modeling in a spreadsheet. A spreadsheet is a format in which a number of cells can be found where each cell is arranged within a worksheet and designated a Row and Column coordinate. The standard nomenclature is that Columns are sequentially lettered and run horizontally and Rows are numbered and run vertically. Thus A1 refers to the cell in the first Column, first Row, cell B3 refers to the cell in the second Column, third Row and so on. Numbers manually entered into a cell can be used in calculations by applying in-cell formulas using in-built mathematical func- 


\begin{tabular}{|c|c|c|c|c|c|c|c|}
\hline & $\mathbf{A}$ & B & C & D & $\mathbf{E}$ & $\mathbf{F}$ & $\mathbf{G}$ \\
\hline 1 & & & & $\mathbf{m}_{\infty}$ & $\mathbf{m}_{\mathbf{0}}$ & $\tau \mathbf{m}$ & $\mathbf{g}$ \\
\hline 2 & V1 & -70 & & 0.00 & 0.00 & 0.80 & -4019.1 \\
\hline 3 & V2 & -25 & & 0.88 & $\mathbf{0 . 0 0}$ & 1.17 & -1435.4 \\
\hline 4 & V3 & -50 & & 0.00 & 0.88 & 1.16 & -2878.2 \\
\hline \multicolumn{8}{|l|}{5} \\
\hline \multicolumn{8}{|l|}{6} \\
\hline \multicolumn{8}{|l|}{7} \\
\hline \multicolumn{8}{|l|}{8} \\
\hline 9 & $t$ (sim) & t (graph) & $\mathrm{i}(\mathbf{p A})$ & & & & \\
\hline 10 & 0.00 & 0.00 & 0 & & & & \\
\hline 11 & 0.01 & 0.01 & $\mathbf{0}$ & & & & \\
\hline 12 & 0.02 & 0.02 & $\mathbf{0}$ & & & & \\
\hline 13 & 0.03 & 0.03 & 0 & & & & \\
\hline 14 & 0.04 & 0.04 & 0 & & & & \\
\hline 15 & 0.05 & 0.05 & $\mathbf{0}$ & & & & \\
\hline & & & & & & & \\
\hline 511 & 0.01 & 5.01 & -0.0 & & & & \\
\hline 512 & 0.02 & 5.02 & -0.3 & & & & \\
\hline 513 & 0.03 & 5.03 & -0.7 & & & & \\
\hline 514 & 0.04 & 5.04 & -1.2 & & & & \\
\hline 515 & 0.05 & 5.05 & -1.9 & & & & \\
\hline & & & & & & & \\
\hline 1511 & 0.01 & 15.01 & -2207.5 & & & & \\
\hline 1512 & 0.02 & 15.02 & -2169.7 & & & & \\
\hline 1513 & 0.03 & 15.03 & -2132.5 & & & & \\
\hline 1514 & 0.04 & 15.04 & -2095.9 & & & & \\
\hline 1515 & 0.05 & 15.05 & -2060.0 & & & & \\
\hline
\end{tabular}

Fig. 2. Spreadsheet template used to simulate the response of a voltage gated ion channel to a step pulse in voltage. The values of $m_{\infty}, m_{\mathrm{o}}, \tau m$ and $g$ calculated in Columns $\mathrm{D}$ to $\mathrm{G}$ from the parameter values in cells $\mathrm{B} 2$, B3 and B4 are used to conduct the simulation by solving for $\mathrm{i}$ in Column C. Rows 16-510 and 516-1510 have been omitted to facilitate viewing of the spreadsheet.

tions. In Excel preceding an argument in a cell by the ' $=$ ' sign designates it as a formula and returns the solution of the formula in the cell. In Fig. 1 the number 5 is entered in cell A1. Typing in the formula ' $=\mathrm{A} 1^{\wedge}{ }^{2}$ ' (quotation marks are used to for illustrative purposes, they are not included in the formula) into cell B1 will result in 25 appearing in B1 (note that in actuality the formula is 'hidden' but the solution to the equation is displayed). If the number in $\mathrm{A} 1$ is changed to 12 then the value of $\mathrm{B} 1$ changes to 144 , as the value returned in $\mathrm{B} 1$ automatically updates to changes in cell A1.

Cell location within a spreadsheet can be referred to as either absolute or relative. Absolute references can be referred to from anywhere in the spreadsheet and refer to a fixed cell location.
Relative references give the reference of a cell relative to a cell where a calculation is being carried out. Column D is configured such that it returns the square of the number in the equivalent Row in Column $\mathrm{C}$ and Column $\mathrm{E}$ is configured such that each cell in Column $\mathrm{E}$ returns the square of the value of cell $\mathrm{C} 1$. Relative references are used when the cell referred to changes with relation to the cell where the calculation is occurring. Absolute references are used when the value of a particular cell is used in calculations in multiple cells.

In Fig. 1 it can be seen that in Columns D and E multiple Rows have been used. Excel has two features that allow multiple Rows to be filled without having to type in the formula for each cell. The first feature is Autofill. Thus in the 


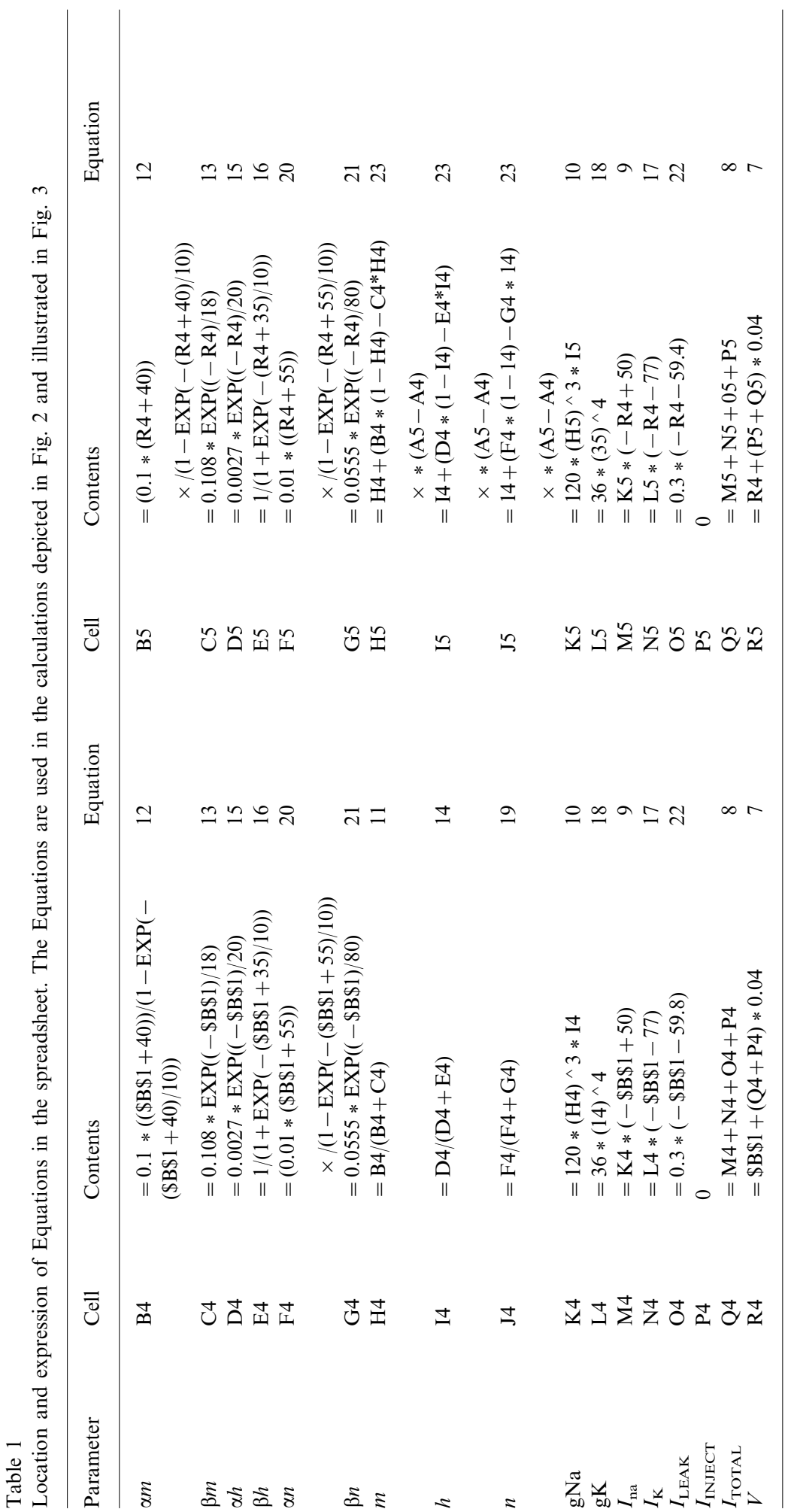


example shown in Fig. 1 cells $\mathrm{C} 1$ to $\mathrm{C} 8$ are filled with values $1-8$, respectively and the formula ' $=\mathrm{A} 11^{\wedge} 2$ ' is typed into cell D1. With this cell highlighted the mouse is placed over the bottom right corner of the cell until a cross appears. This is called the fill handle. The left hand mouse button is held down, the mouse dragged down to Row 8 and then released. The cells in D1 to D8 will have automatically filled with the formulas as shown. The formula ' $=\$ C \$ 1^{\wedge} 2^{\prime}$ is typed into cell E1. Prefacing the Column and Row values with the ' $\$$ ' sign designates it as an absolute reference. Dragging the mouse as described above down to cell E8 causes the cells E2 to E8 to fill with the same
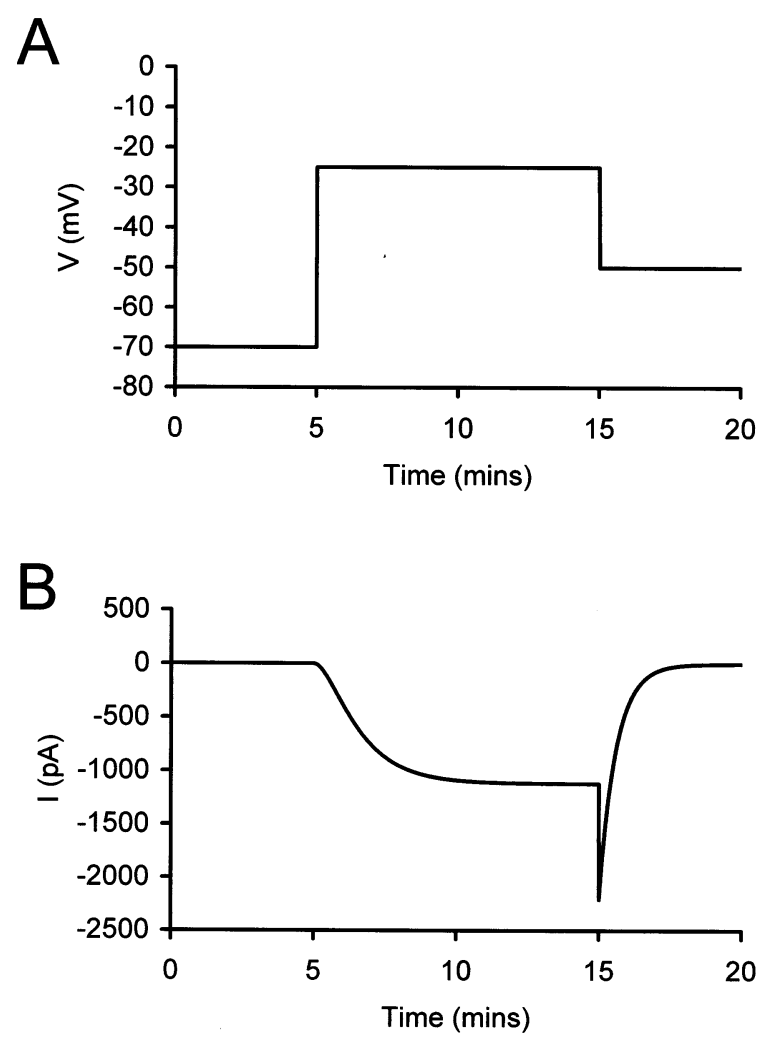

Fig. 3. Illustration of the simple simulation. (A) The voltage steps from -70 to $-25 \mathrm{mV}$ for $10 \mathrm{~ms}$ then returns to -50 $\mathrm{mV}$ for $5 \mathrm{~ms}$. (B) The voltage gated ion channels response to the voltage protocol shown in A. Current is at a steady state of $0 \mathrm{pA}$ for the initial $5 \mathrm{~ms}$ at $-70 \mathrm{mV}$, then increases in amplitude with the typical sigmoidal onset indicative of the activation parameter raised to the power 2, before reaching steady state. Return of the voltage to $-50 \mathrm{mV}$ resulted in a large tail current that decayed mono-exponentially to $0 \mathrm{pA}$. formula (' $\left.=\$ C \$ 1^{\wedge} 2^{\prime}\right)$ as the reference is absolute and does not change, irrespective of the location of the cell referring to it.

The second feature that helps with filling cells is the fill down tool. This tool is not automatically displayed in Excel and must be installed on the tool bar at the top of the worksheet. This is done by clicking on the View menu and selecting Customize under Toolbars. Click the Commands tab and the fill down tool (white downward facing arrow on a dark blue background) is the tenth tool down in the Edit category. Placing the cursor on the tool and holding down the left hand mouse button and dragging it onto the toolbar at the top of the worksheet permanently places this tool on the toolbar. This tool copies the contents and formats of the topmost cells of a selected range into the cells below. For example if the cube value in Column D is wanted it would be tedious to change this by typing ' $=\mathrm{C}^{* \wedge} 3^{\text {' }}$ (* depicts the Row number) into the cells D1 to D8. To automatically change the formula in all the cells begin by typing the formula ' $=\mathrm{C} 1{ }^{\wedge} 3$ ' in cell D1. Then with the cell D1 highlighted hold down both the Control and Shift buttons and press the down arrow on the keyboard. The cells D1 to D8 will be highlighted. Press the fill down toolbar and all the formulas will automatically change to ' $=\mathrm{C}^{* \wedge} 3^{\text {' }}$ as the formula has been copied into all the highlighted cells.

\subsection{Configuring spreadsheet for simple simulation}

The model used to illustrate a simple simulation is the response of a high voltage activated calcium channel to a voltage step to $-25 \mathrm{mV}$ (V2) for 10 $\mathrm{ms}$ from a holding potential of $-70 \mathrm{mV}$ (V1) as defined by the following relationship (see Eq. (1) in the Appendix for details):

$i=\left(\left(m_{\infty}-m_{\mathrm{o}}\right) * \exp \frac{-t}{\tau m}\right)^{2} * g$

The voltage is then changed to $-50 \mathrm{mV}$ (V3) for $5 \mathrm{~ms}$. The values of the voltages are typed into cell B2, B3 and B4, respectively, as illustrated in Fig. 2. Cells A2, A3 and A4 are labeled V1, V2 and V3, respectively, to describe the cells. In Columns D to $\mathrm{G}$ the values of $m_{\infty}, m_{\mathrm{o}}, \tau m$ and $g$ for the voltages V1 (Row 2), V2 (Row 3) and V3 


\begin{tabular}{|c|c|c|c|c|c|c|c|c|c|c|c|c|c|c|c|c|c|c|}
\hline & A & B & C & $D$ & $\mathbf{E}$ & $F$ & G & H & I & $\mathbf{J}$ & K & $\mathbf{L}$ & M & $\mathbf{N}$ & 0 & $\mathbf{P}$ & $\mathbf{Q}$ & $\mathbf{R}$ \\
\hline 1 & $\bar{v}_{\mathrm{i}}$ & -70 & & & & & & & & & & & & & & & & \\
\hline 2 & $I_{\text {INJECT }}$ & 10 & & & & & & & & & & & & & & & & \\
\hline 3 & time (ms & $\alpha \mathbf{m}$ & $\beta m$ & $\alpha h$ & $\beta \mathbf{h}$ & $\alpha \mathbf{n}$ & $\beta \mathbf{n}$ & $\mathbf{m}$ & $h$ & $\mathbf{n}$ & $\mathrm{gNa}$ & gK & $I_{\mathrm{Na}}$ & $I_{K}$ & ILEAK & JECT & I TOTAL & $\mathbf{v}$ \\
\hline 4 & 0.00 & 0.16 & 5.28 & 0.09 & 0.03 & 0.04 & 0.13 & 0.03 & 0.75 & 0.24 & 0.00 & 0.13 & 0.26 & \begin{tabular}{|l|}
-0.90 \\
\end{tabular} & 3.06 & .00 & 2.42 & -69.98 \\
\hline 5 & & \begin{tabular}{|l|}
0.16 \\
\end{tabular} & 5.27 & 09 & 0.03 & 0.04 & 0.13 & 0.03 & 0.75 & 0.24 & 0.00 & 0.13 & 0.26 & $\mid-0.90$ & 3.17 & .00 & 2.53 & -69.87 \\
\hline 6 & .08 & 0.16 & 5.24 & 0.09 & 0.03 & 0.04 & 0.13 & 0.0 & 0.75 & 0.24 & 0.00 & 0.13 & & & 3.14 & 0.00 & 2.49 & -69.77 \\
\hline 7 & 0.12 & 0.16 & 5.21 & .09 & 0.03 & 0.04 & 0.13 & 0.03 & 0.75 & 0.24 & 0.00 & 0.13 & 0.27 & \begin{tabular}{|l|}
-0.93 \\
\end{tabular} & 3.11 & 0.00 & 2.45 & -69.68 \\
\hline 8 & 16 & 0.16 & 5.18 & 0.09 & 0.03 & 0.04 & 0.13 & 0.03 & 0.75 & 0.24 & 0.00 & 0.13 & 0.27 & -0.94 & 3.08 & .00 & 2.41 & -69.58 \\
\hline 9 & 0 & \begin{tabular}{|l|}
0.16 \\
\end{tabular} & 5.16 & .09 & 0.03 & 0.04 & 0.13 & 0.03 & 0.75 & 0.24 & 0.00 & 0.13 & 0.27 & \begin{tabular}{|l|}
-0.96 \\
\end{tabular} & 3.05 & 0.00 & 2.37 & -69.49 \\
\hline 10 & 0.24 & 0.16 & 5.13 & 0.09 & 0.03 & 0.04 & 0.13 & 0.03 & 0.75 & 0.24 & 0.00 & 0.13 & 0.28 & \begin{tabular}{|l|}
-0.97 \\
\end{tabular} & 3.03 & 00 & 2.34 & -69.39 \\
\hline 11 & 28 & 0.16 & 5.10 & 0.09 & 0.03 & 0.04 & 0.13 & 0.03 & 0.75 & 0.24 & 0.00 & 0.13 & 0.29 & -0.98 & 3.00 & 0.00 & 2.30 & -69.30 \\
\hline 12 & & 0.17 & 5.08 & 0.09 & 0.03 & 0.04 & 0.13 & 0.03 & 0.75 & 0.24 & 0.00 & 0.13 & 0.30 & \begin{tabular}{|l|}
-0.99 \\
\end{tabular} & 2.97 & 0.00 & 2.27 & -69.21 \\
\hline 13 & 36 & 0.17 & 5.05 & 0.09 & 0.03 & 0.05 & 0.13 & 0.03 & 0.75 & 0.24 & 0.00 & 0.13 & 0.30 & \begin{tabular}{|l|}
-1.01 \\
\end{tabular} & 2.94 & 0.00 & 2.24 & -69.12 \\
\hline & & & & & & & & & & & & & & & & & & \\
\hline 504 & & 0.20 & 4.34 & .07 & 04 & 05 & .13 & 04 & 6 & 0.29 & 0.01 & .27 & 0.79 & -2.86 & 2.12 & 0.00 & 0.06 & -66.08 \\
\hline 505 & & 0.21 & 4.24 & 07 & 0.04 & 0.05 & 0.13 & 0.04 & 0.65 & 0.29 & 0.01 & 0.27 & 0.79 & -2.97 & 2.00 & 10.00 & -0.17 & -65.68 \\
\hline 506 & 20.08 & \begin{tabular}{|l|}
0.21 \\
\end{tabular} & 4.15 & 0.07 & 0.04 & 0.06 & 0.13 & 0.04 & 0.65 & 0.29 & 0.01 & 0.27 & 0.81 & -3.08 & 1.89 & 10.00 & -0.38 & -65.30 \\
\hline 507 & 20.12 & 0.22 & 4.06 & 0.07 & 0.05 & 0.06 & 0.13 & 0.05 & 0.65 & 0.29 & 0.01 & 0.27 & 0.84 & -3.18 & \begin{tabular}{|l|}
1.77 \\
\end{tabular} & 10.00 & -0.57 & -64.92 \\
\hline 508 & 20.16 & 0.22 & 3.98 & 0.07 & 0.05 & 0.06 & 0.12 & 0.05 & 0.65 & 0.29 & 0.01 & 0.27 & 0.90 & -3.29 & 1.66 & 10.00 & -0.74 & -64.55 \\
\hline 509 & & 0.23 & 3.90 & 0.07 & 0.05 & 0.06 & 0.12 & 0.05 & 0.65 & 0.30 & 0.01 & 0.27 & 0.96 & -3.40 & 1.55 & 10.00 & -0.89 & -64.19 \\
\hline 510 & & 0.24 & 3.82 & 0.07 & 0.05 & 0.06 & 0.12 & 0.05 & 0.65 & 0.30 & 0.01 & 0.27 & 1.04 & -3.51 & \begin{tabular}{|l|l|}
1.44 \\
\end{tabular} & 10.00 & -1.03 & -63.83 \\
\hline 511 & 20.28 & 0.24 & 3.74 & 0.07 & 0.05 & 0.06 & 0.12 & 0.05 & 0.65 & 0.30 & 0.01 & 0.28 & 1.14 & -3.62 & 1.33 & 0.00 & -1.15 & -63.47 \\
\hline 512 & & 0.25 & 3.67 & 0.06 & 0.05 & 0.06 & 0.12 & 0.05 & 0.65 & 0.30 & 0.01 & 0.28 & 1.25 & -3.73 & 1.22 & 10.00 & -1.26 & -63.12 \\
\hline 513 & 20.36 & 0.25 & 3.60 & 0.06 & 0.06 & 0.06 & 0.12 & 0.05 & 0.65 & .30 & 0.01 & 0.28 & 1.37 & -3.85 & 1.12 & 10.00 & -1.36 & -62.78 \\
\hline
\end{tabular}

Fig. 4. Spreadsheet template used to calculate $V$. The value for $V_{\mathrm{i}}$ in cell $\mathrm{B} 1$ is used in the initial calculations in Row 4 . The value of $V$ in cell R4 ( $\left.V_{\text {old }}\right)$ is then used as the voltage parameter in the calculations in Row 5 to calculate $V_{\text {new }}$. Calculations are carried out sequentially down the Rows where the value for $V$ calculated in Column $\mathrm{R}$ of the previous Row is used as $V_{\text {old }}$ in the calculations of the subsequent Row to calculate $V_{\text {new }}$.

(Row 4) are calculated using in-cell formulas. Firstly cells D1, E1, F1 and G1 are labeled to indicate which variable is being calculated in each Column. The value of $m_{\infty}$ at V1 is calculated in cell D2. The formula

$$
=1 /(1+\exp (-24.6-\$ B \$ 2) / 11.3)
$$

is entered into cell D2. This is Eq. (2) (see Appendix) rearranged in a form recognized by Excel. This formula is also entered into cell E2, as the values of $m_{\infty}$ and $m_{\mathrm{o}}$ are the same at V1. Note that $\$ B \$ 2$ is an absolute reference.

The value of $\tau m$ (Eq. (3); Appendix) at V1 is calculated by entering

$$
=1.25 *(1 / \cosh (-0.031 *(\$ B \$ 2+37.1)))
$$

in cell F2 and in a similar fashion $g$ (Eq. (4); Appendix) is calculated using the formula

$$
=-57.4 * \$ \mathrm{~B} \$ 2 /(\exp (0.117 * \$ \mathrm{~B} \$ 2)-1)
$$

in cell $\mathrm{G} 2$.
The same method is used to calculate the values of $m_{\infty}, m_{\mathrm{o}}, \tau m$ and $g$ at the subsequent voltages V2 and V3. Table 1 illustrates the location and contents of the cells shown in Fig. 2. To complete the simulation the values of solutions to Eqs. (2)-(4) described above are input into Eq. (1). Columns A and B contain data pertaining to time and Column $\mathrm{C}$ is used to solve $i$. The time data in Column $\mathrm{A}$ is used to conduct the simulation and the time data in Column B is used to chart the data. Firstly cells A9, B9 and C9 are labeled $t$ (sim), $t$ (graph) and $i(\mathrm{pA})$. Column $\mathrm{A}$ is filled from Row 10-510 starting at 0.00 in cell A10 and increasing in $0.01 \mathrm{~ms}$ increments for the first $5 \mathrm{~ms}$ of the simulation where the voltage is $-70 \mathrm{mV}$ (V1). The Autofill method is used to fill down the Rows to cell A510. At Row 5110.01 is entered into A511 as the calculations require the voltage to be reset to zero at each voltage change, 0.02 is entered in A512 and this Row is filled down to 
Table 2

Location and expression of Equations used in the calculations in Rows 4 and 5 of the complex simulation

\begin{tabular}{|c|c|c|c|}
\hline Parameter & Cell & Contents & Equation \\
\hline V1 & $\mathrm{B} 2$ & -70 & - \\
\hline $\mathrm{V} 2$ & B3 & -25 & - \\
\hline $\mathrm{V} 3$ & B4 & -50 & - \\
\hline$m_{\infty}(\mathrm{V} 1)$ & D2 & $=1 /(1+\operatorname{EXP}(-24.6-\$ B \$ 2) / 11.3)$ & 2 \\
\hline$m_{\mathrm{o}}(\mathrm{V} 1)$ & E2 & $=1 /(1+\operatorname{EXP}(-24.6-\$ B \$ 2) / 11.3)$ & 2 \\
\hline$g(\mathrm{~V} 1)$ & G2 & $=-57.4 * \$ \mathrm{~B} \$ 2 /(\mathrm{EXP}(0.117 * \$ \mathrm{~B} \$ 2)-1)$ & 4 \\
\hline$m_{\infty}(\mathrm{V} 2)$ & D3 & $=1 /(1+\operatorname{EXP}(-24.6-\$ B \$ 3) / 11.3)$ & 2 \\
\hline$m_{\mathrm{o}}(\mathrm{V} 2)$ & E3 & $=1 /(1+\operatorname{EXP}(-24.6-\$ B \$ 2) / 11.3)$ & 2 \\
\hline$\tau m(\mathrm{~V} 2)$ & F3 & $=1.25 *(1 / \mathrm{COSH}(-0.031 *(\$ \mathrm{~B} \$ 3+37.1)$ & 3 \\
\hline$g(\mathrm{~V} 2)$ & G3 & $=-57.4 * \$ \mathrm{~B} \$ 3 /(\mathrm{EXP}(0.117 * \$ \mathrm{~B} \$ 3)-1)$ & 4 \\
\hline$g(\mathrm{~V} 3)$ & G4 & $=-57.4 * \$ \mathrm{~B} \$ 4 /(\mathrm{EXP}(0.117 * \$ \mathrm{~B} \$ 4)-1)$ & 4 \\
\hline$i$ & $\mathrm{C} 10$ & $=(\$ D \$ 2-(\$ D \$ 2-\$ E \$ 2) * \operatorname{EXP}(-\mathrm{A} 10 / \$ F \$ 2))^{\wedge} 2 * \$ G \$ 2$ & 1 \\
\hline$i$ & C511 & $=(\$ D \$ 3-(\$ D \$ 3-\$ E \$ 3) * \operatorname{EXP}(-\mathrm{A} 511 / \$ F \$ 3))^{\wedge} 2 * \$ G \$ 3$ & 1 \\
\hline$i$ & $\mathrm{C} 1511$ & $=(\$ D \$ 4-(\$ D \$ 4-\$ E \$ 4) * \operatorname{EXP}(-\mathrm{A} 1511 / \$ F \$ 4))^{\wedge} 2 * \$ G \$ 4$ & 1 \\
\hline
\end{tabular}

See text for details.

A1510 representing the $10 \mathrm{~ms}$ voltage step to V2. Finally A1511-A2010 are filled from 0.01 to 5.00 in $0.01 \mathrm{~ms}$ steps for the final $5 \mathrm{~ms}$ of the simulation when the cell is at $-50 \mathrm{mV}$ (V3). Column B is filled from B10 starting at 0.00 and incrementing in 0.01 steps to $20.00 \mathrm{~ms}$ in B2010 using Autofill. To calculate the current (i) in cell C10 the formula

$$
\begin{aligned}
= & (\$ D \$ 2-(\$ D \$ 2-\$ E \$ 2) \exp (-\mathrm{A} 10 / \$ \mathrm{E} \$ 2)) \\
& \hat{\times} 2 * \$ \mathrm{G} \$ 2
\end{aligned}
$$

is entered. This is Eq. (1) (Appendix). Note that all the voltage dependent parameters references are absolute, referring to the values calculated in the above section, only the time reference is relative. This Column is filled to A510. At cell C511 the formula

$$
\begin{aligned}
= & (\$ D \$ 3-(\$ D \$ 3-\$ E \$ 3) \exp (-\mathrm{A} 510 / \$ E \$ 3)) \\
& \hat{\times} 2 * \$ G \$ 3
\end{aligned}
$$

is entered. This contains data for the step voltage to V2. Rows C511 to C1510 are filled.
The formula

$$
\begin{aligned}
= & (\$ D \$ 4-(\$ D \$ 4-\$ E \$ 4) \exp (-\mathrm{A} 1510 / \$ E \$ 4)) \\
& \hat{\times} 2 * \$ G \$ 4
\end{aligned}
$$

is entered in cell C1511 and filled down to C2010 for the $5 \mathrm{~ms}$ period at V3. The completed simulation is illustrated (Fig. 3) by charting Column B as the $x$-axis and Column $\mathrm{C}$ as the $y$-axis.

\subsection{Configuring spreadsheet for complex simulation}

The preparation used to demonstrate current clamp simulations is the squid giant axon containing $I_{\mathrm{Na}}, I_{\mathrm{K}}$ and $I_{\mathrm{LEAK}}$. Fig. 4 illustrates the spreadsheet template used in the simulation. Cell B1 contains the initial voltage $\left(V_{\mathrm{i}}\right)$ and cell $\mathrm{B} 2$ contains the injected current $\left(I_{\text {INJECT }}\right)$. Column A contains the time parameter where the time increment is $0.04 \mathrm{~ms}$ and the duration of the simulation is $160 \mathrm{~ms}$. Columns $\mathrm{B}$ to $\mathrm{R}$ contain equations used in the simulation. Row 4 contains the equations used to conduct the initial part of the simulation. This involves using $V_{\mathrm{i}}$ as $V_{\text {old }}$ (Eq. (7)) in the formulae in cells B4 to Q4 to calculate the new 
value of $V\left(V_{\text {new }}-\right.$ Eq. (7)) in cell R4. Table 2 depicts the contents of the cells in Row 4 and the Equations upon which the in-cell formulae are based. The value of $V$ in $\mathrm{R} 4$ is used in the formulae in Row 5 to calculate the value of $V$ in cell R5. Thus $V$ in the previous Row ( $\left.V_{\text {old }}\right)$ is used in the formulae in the subsequent Row to calculate the new value of $V\left(V_{\text {new }}\right)$. This is shown in Table 2 where the contents of Row 5 use R4 as the value for $V$. Note also that Eqs. (11), (14) and (19) are used to calculate the initial values of $m, n$ and $h$, respectively, in Row 4 , but in all subsequent Rows Eq. (23) is used. The time in Column A is filled down to Row 4004 and the formulae in Rows $\mathrm{B}$ to $\mathrm{R}$ are filled down from Row 5 by highlighting the block of cells from B5 to R5. The cursor is placed over the bottom right hand cor-
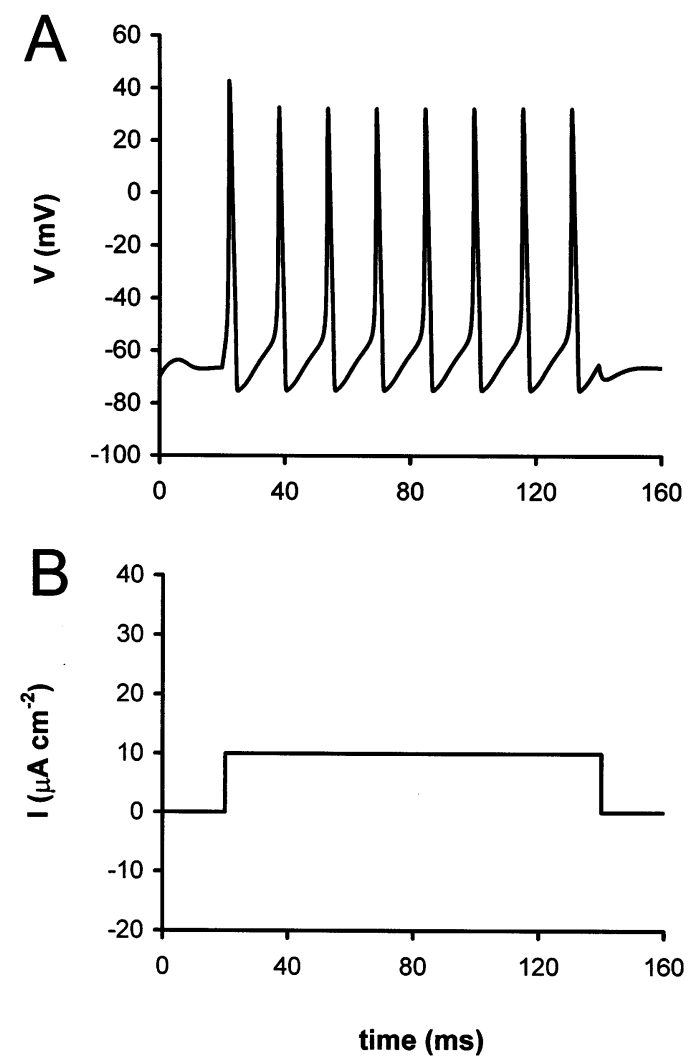

Fig. 5. Illustration of the complex simulation. An injection of a current pulse of $10 \mu \mathrm{A}$ for $120 \mathrm{~ms}$ starting $20 \mathrm{~ms}$ into the simulation (B) results in regenerative firing of action potentials (A) which continues for the duration of the current injection. ner of cell R4 and the fill handle is dragged vertically downwards to cell R4004 by holding down the left-hand mouse button. This fills in all the cells in the block containing B5 to R4004. Finally the duration and amplitude of the injected current is entered in Column P. In the example shown cells P504 to P3504 contain the formula ' $=$ \$B $\$ 2$ '. The other cells in the Column contain 0 . This completes the simulation and the result is illustrated in Fig. 5.

\section{Discussion}

One of the purposes in modeling biological systems is to use experimentally obtained data to produce an accurate model of a system which can subsequently be used to forecast unpredicted behavior. In the case of the current clamp simulation illustrated in this study modeling can be used to predict the contribution of individual ionic currents to overall firing activity by removing that particular current from the simulation, a procedure that may be impossible experimentally. The level of complexity in producing this model is not great and this study demonstrates the feasibility of carrying out solutions on standard equipment available to most biologists. For example the voltage clamp simulation would be suitable to conduct a simulation of voltage gated calcium channels [5] and the current clamp simulation would be suitable to simulate the firing activity in a neuron [6].

A basic knowledge of spreadsheet function is necessary but the more complicated programming with the in-built macro language is not needed to implement the methodology described. The use of Excel in the present context is as a convenient input/output interface between insertion of the data and the result of the simulation. The methodology should be comparable to a simulation carried out on specialized software as long as the model equations are solved in an identical manner. Programs use looping structures where data is calculated in a cyclical manner before proceeding to the next stage of the calculation. Excel uses an identical procedure with the exception that the product of the calculations is dis- 
played in the spreadsheet and not hidden within the workings of the program. This makes debugging easier in Excel as the result of the calculations is visible and any non-sensical values can be easily identified. In cyclical types of calculations errors in the initial stages are carried on through the entire simulation. Excel has a useful feature where in-cell formulas are color coded such that each cell referred to in a formula is highlighted with the same color as the expression in the formula making identification of cells referred to within formulas easy. This facilitates debugging of the simulation. Once the correct formula has been initially entered the process of copying formulas using Autofill ensures that the in-cell formulas at least are error free.

The methodology is also very flexible and fast to calculate and chart data. For example it is a simple procedure to alter the time increment of the simulation, simply enter the new time increment in Row 2 of the simulation and copy down all the rows. Likewise either adding additional Rows using Autofill or deleting Rows of data can be used to alter the duration of the simulation. Although it requires 64000 sequential calculations and the charting of the data the current clamp simulation described in this paper takes less than $1 \mathrm{~s}$ to run on a Pentium $133 \mathrm{MHz}$ computer with $32 \mathrm{MB}$ of RAM.

\section{Appendix A}

This appendix offers a very brief overview of the calculations used in both voltage clamp and current clamp simulations. For a description of voltage clamp and current clamp simulations see [7-9].

\section{A.1. Voltage clamp simulation}

The first example used in the paper is the response of a neuron containing high voltage activated calcium channels under voltage clamp conditions [7]. The voltage is held initially at -70 $\mathrm{mV}$ (V1) for $5 \mathrm{~ms}$ and is then changed to -25 $\mathrm{mV}$ (V2) for $10 \mathrm{~ms}$ before returning to $-50 \mathrm{mV}$ (V3) for $5 \mathrm{~ms}$. The model uses the Hodgkin
Huxley series of equations [9] which describe the activation of voltage gated ion channels. The channel is said to be in a steady state when the voltage is constant but when the voltage changes the current (i) reaches a new steady described by:

$i=\left(m_{\infty}-\left(m_{\infty}-m_{\mathrm{o}}\right) * \exp \frac{-t}{\tau m}\right)^{2} * g$

where $m$ is the voltage dependent activation parameter that governs the opening of the channel, $g$ is the instantaneous $\mathrm{I}-\mathrm{V}$ relationship of the current which describes how much current will flow through an open channel at a particular voltage and $\tau m$ is the time constant that governs the rate of opening and closing of the channel.

The activation parameter $(m)$ is described by the Boltzmann relation:

$m=\left[1+\exp \frac{(-24.6-V)}{11.3}\right]$

where $V$ is the voltage. When the voltage is stepped from one voltage to a new voltage the equation predicts that $m$ relaxes exponentially to its new value, where $m_{\infty}$ is the new steady state value of $m$ and $m_{\mathrm{o}}$ is the value of $m$ at the previous voltage.

The time constant $\tau \mathrm{m}$ is described by:

$\tau m=1.25 \frac{1}{\cosh (-0.031(V+37.1))}$

The instantaneous $\mathrm{I}-\mathrm{V}$ relation is described by:

$g=-57.4(\exp (0.116) * V)$

\section{A.2. Current clamp simulation}

In the Hodgkin Huxley model of a squid giant axon [9] containing $I_{\mathrm{Na}}, I_{\mathrm{K}}$ and $I_{\mathrm{LEAK}}$ the change in voltage in response to injected current is given by:

$\frac{\mathrm{d} V}{\mathrm{~d} t}=\frac{I_{\mathrm{TOTAL}}}{C}$

which can be rewritten as

$V_{\text {new }}=V_{\text {old }}+\frac{I_{\mathrm{TOTAL}}^{*} \delta t}{C}$ 
as $C$ is the membrane capacitance and is assumed to be $1 \mu \mathrm{F} \mathrm{cm}^{-2}$ this can be reduced to

$$
V_{\text {new }}=V_{\text {old }}+\left(I_{\text {TOTAL }}^{*} \delta t\right)
$$

$\delta t$ is the time increment and $I_{\mathrm{TOTAL}}$ is the total trans-membrane current. Thus:

$I_{\mathrm{TOTAL}}=I_{\mathrm{Na}}+I_{\mathrm{K}}+I_{\text {leak }}+I_{\mathrm{INJECT}}$

$I_{\mathrm{Na}}$ is calculated using Eqs. (9)-(16)

$I_{\mathrm{Na}}=g_{\mathrm{Na}}(-V+50) \mu \mathrm{A} \mathrm{cm}^{-2}$

$g_{\mathrm{Na}}=120 \mathrm{~m}^{3} \mathrm{~h} \mathrm{~S} \mathrm{~cm}{ }^{-2}$

$m=\frac{\alpha_{m}}{\alpha_{m}+\beta_{m}}$

$\alpha_{m}=\frac{0.1(V+40)}{1-\exp \left[\frac{-(V+40)}{10}\right]}$

$\beta_{m}=0.108 \exp \frac{-V}{18}$

$h=\frac{\alpha_{h}}{\alpha_{h}+\beta_{h}}$

$\alpha_{h}=0.0027 \exp \frac{-V}{20}$

$\beta_{h}=\frac{1}{1+\exp \left[\frac{-(V+35)}{10}\right]}$

$I_{\mathrm{K}}$ is calculated using Eqs. (17)-(21):

$$
\begin{aligned}
& I_{\mathrm{K}}=g_{\mathrm{K}}(-V-77) \mu \mathrm{A} \mathrm{cm}^{-2} \\
& g_{\mathrm{K}}=36 \mathrm{n}^{4} \mathrm{~S} \mathrm{~cm}^{-2} \\
& n=\frac{\alpha_{n}}{\alpha_{n}+\beta_{n}} \\
& \alpha_{n}=\frac{0.01(V+55)}{1-\exp \left[\frac{-(V+55)}{10}\right]}
\end{aligned}
$$

$\beta_{n}=0.0555 \exp \frac{-V}{80}$

and finally

$I_{\text {LEAK }}=0.3(-V-59.4) \mu \mathrm{A} \mathrm{cm}^{-2}$

In this simulation the Hodgkin Huxley parameters do not reach steady state and Euler integration

$m=\alpha m+\left(\alpha m *\left(1-m_{\mathrm{o}}\right)-\beta m * m_{\mathrm{o}}\right) * \delta t$

is used [10] to calculate the values of $m, h$ and $n$.

\section{References}

[1] E. De Schutter, A consumer guide to neuronal modeling software, Trends Neurosci. 15 (1992) 462-464.

[2] P. Revest, Neurosim for Windows, Trends Neurosci. 18 (1995) 556.

[3] M.L. Hines, N.T. Carnevale, The NEURON simulation environment, Neural Comput. 9 (1997) 1179-1209.

[4] J.M. Bower, D. Beeman, The Book of Genesis, Springer, Berlin, 1995.

[5] J.G.G. Borst, B. Sakmann, Calcium current during a single action potential in a large presynaptic terminal of the rat brainstem, J. Physiol. 506 (1998) 143-157.

[6] C.C. Canavier, J.W. Clark, J.H. Byrne, Simulation of the bursting activity of neuron R15 in Aplysia: role of ionic currents, calcium balance and modulatory transmitters, J. Neurophysiol. 66 (1991) 2107-2124.

[7] A.M. Brown, P.C. Schwindt, W.E. Crill, Voltage dependence and activation kinetics of pharmacologically defined components of the high threshold calcium current in rat neocortical pyramidal cells, J. Neurophysiol. 70 (1993) 1530-1543.

[8] B. Hille, Ionic basis of resting and action potentials, in: E. Kandel (Ed.), Handbook of Physiology, American Physiological Society, Bethesda, MD, 1977, pp. 99-136.

[9] A.L. Hodgkin, A.F. Huxley, A quantitative description of membrane current and its application to conduction and excitation in nerve, J. Physiol. 117 (1952) 500-544.

[10] J.W. Moore, F. Ramon, On numerical integration of the Hodgkin and Huxley equations for a membrane action potential, J. Theor. Biol. 45 (1974) 249-273. 